\title{
artigo
}

\section{Tratamento na atenção básica de leishmaniose cutânea com apliç̧ão intralesional de glucantime em região da face}

\author{
Treatment in basic attention of leshimaniosis with intralesional application of glucantime in face region
}

Tratamiento en la atención básica de leshimaniosis con aplicación intralesional de glucantime en región facial

\begin{abstract}
RESUMO
A leishmaniose tegumentar americana é uma doença infecciosa endêmica na saúde pública do país. A doença é transmitida após a picada das fêmeas do mosquito hematófago, conhecido como flebotomíneo e possui diversas manifestações clínicas, classificadas em cutânea, cutaneomucosa e cutânea difusa, acometendo todas os gêneros e faixas etárias. 0 objetivo desse artigo é relatar o caso clínico de um paciente, apresentando lesão ulcerada em região da face. 0 método utilizado foi a identificação de Leishmania através aa reação em cadeia da polimerase associada a resposta clínica ao tratamento após aplicação intralesional. Como resultado foram obtidas respostas imediatas das lesões que apesar de estudos apresentarem cura espontaneamente após meses/anos, lesões faciais necessitam de um protocolo mais agressivo. Conclui-se que a importância do relato se deve ao fato do tratamento terapêutico consistir a aplicação direta em local não liberado pelo protocolo do Ministério da Saúde de leishmaniose tegumentar americana.
\end{abstract}

DESCRITORES: Leishmaniose; Ferimentos e Lesões; Antimoniato de Meglumina.

\section{ABSTRACT}

American cutaneous leishmaniasis is an endemic infectious disease in the country's public health. The disease is transmitted after the bite of females of the hematophagous mosquito, known as sand fly, and has several clinical manifestations, classified as cutaneous, cutaneous-mucosal and diffuse cutaneous, affecting all genders and age groups. The aim of this article is to report the clinical case of a patient, presenting an ulcerated lesion in the region of the face. The method used was the identification of Leishmania through the polymerase chain reaction associated with clinical response to treatment after intralesional application. As a result, immediate responses were obtained from the lesions that, despite studies showing spontaneous healing after months/ years, facial lesions need a more aggressive protocol. It is concluded that the importance of the report is due to the fact that the therapeutic treatment consists of direct application in a location not authorized by the protocol of the Ministry of Health for American tegumentary leishmaniasis.

DESCRIPTORS: Leishmaniasis; Wounds and Injuries; Meglumine Antimoniate.

\section{RESUMEN}

La leishmaniasis cutánea americana es una enfermedad infecciosa endémica en la salud pública del país. La enfermedad se transmite tras la picadura de hembras del mosquito hematófago, conocido como flebótomos, y tiene varias manifestaciones clínicas, clasificadas en cutáneas, cutáneo-mucosas y cutáneas difusas, afectando a todos los géneros y grupos de edad. El objetivo de este artículo es reportar el caso clínico de un paciente que presenta una lesión ulcerada en la región de la cara. El método utilizado fue la identificación de Leishmania a través de la reacción en cadena de la polimerasa asociada a la respuesta clínica al tratamiento después de la aplicación intralesional. Como resultado, se obtuvieron respuestas inmediatas de las lesiones que, a pesar de que los estudios muestran una curación espontánea después de meses / años, las lesiones faciales necesitan un protocolo más agresivo. Se concluye que la importancia del informe se debe a que el tratamiento terapéutico consiste en la aplicación directa en un lugar no autorizado por el protocolo del Ministerio de Salud para la leishmaniasis tegumentaria americana. DESCRIPTORES: Leishmaniasis; Heridas y Traumatismos; Antimoniate de méglumine.

RECEBIDO EM: 31/03/2021 APROVADO EM: 17/06/2021

\section{Paula Layana Vieira Wanderley}

Especialista em Saúde da Família pela Universidade Federal do Rio Grande do Norte-UFRN (2020) Graduada em Medicina pela Faculdade de Ciências Biomédicas de Cacoal/RO-FACIMED (2018). Atualmente atua como Médica da Atenção Básica e na área da Clínica Médica.

ORCID: 0000-0002-4707-7529 


\section{Carlos Eduardo Tortelli Veloso}

Especialista em Saúde da Família pela Universidade Federal do Rio Grande do Norte-UFRN (2020) Graduado em Medicina pela Faculdade de Ciências Biomédicas de Cacoal/RO-FACIMED (2018). Atualmente atua como Médico da Atenção Básica e na área da Clínica Médica.

ORCID: 0000-0003-1542-2858

\section{Tâmara Vieira Monção}

Graduanda em Medicina Universidade Federal de Roraima, UFRR (10 semestre). ORCID: 0000-0002-7168-365X

\section{INTRODUÇÃO}

A leishmaniose é uma doença infecto-parasitária crônica com etiologia em parasitas do gênero Leishmania, classificada como uma doença de característica tropical, apresentando-se através de diversas formas clínicas, conforme a espécie do protozoário. Apresentam um ciclo heteroxênico e parasitam insetos vetores e animais vertebrados, onde nos mamíferos contraem a forma de amastigota, imóvel em formato redondo que se replicam dentro das células do sistema fagocitário espalhando-se pelo sistema sanguíneo causando erupções cutâneas, lesionando o tecido epitelial ${ }^{1}$.

As leishmanioses são consideradas um grande problema de saúde pública no mundo, em continentes como a América, Ásia, África e Europa. Constituindo uma endemia com prioridade para controle elevada, e no Brasil, representa relevante papel nos serviços médicos que são portas de entrada para o sistema de saúde públi$\mathrm{co}^{2}$. A Organização Mundial da Saúde (OMS) afirma que aproximadamente 350 milhões de pessoas estão inseridas ao risco de contrair essa infecção e que, ao menos 2 milhões de pessoas configuram novos casos todos os anos, quando traçado um perfil epidemiológico da manifestação da doença pelo mundo 3 .

A doença é considerada como uma das seis mais significativas doenças infecciosas pelo mundo, ficando em segundo lugar, justificada a posição pelo elevado coeficiente de detecção e capacidade de produzir diversos sinais e sintomas de alerta, principalmente as lesões ulcerosas que aparecem em todas as partes do corpo. No Brasil tem sido verificado a presença de endemia, com predomínio de áreas rurais e ambientes provenientes de matas e rios ${ }^{3}$.

Compreende um grupo de doenças infecciosas que podem ocorrer com envolvimento cutâneo ou visceral. A leishmaniose tegumentar americana (LCA), manifestaçôes cutâneas no Novo Mundo, apresenta clinicamente lesões com uma diversidade de variáveis, que vão desde o tipo acneiforme até úlceras com ou sem linfadenopatia. A lesão cutânea típica se apresenta unicamente como úlcera com bordas infiltradas em áreas expostas do corpo, porém a manifestação clínica pode variar dependendo conforme o estado imunológico do hospedeiro, da carga do parasita e dos tipos de espécies de Leishmania envolvidas 4 .

A úlcera típica da leishmaniose cutânea (LC) pode evoluir para a cura em um período de vários meses a anos, se não for diagnosticada e tratada pode causar deformidades e causar até mesmo necrose dos tecidos 5 . Entretanto alguns pacientes apresentam progressão espontânea precoce da doença, são diagnosticados através de exames laboratoriais e critérios clínicos, podendo até mesmo antes do início da terapia medicamentosa, as lesões iniciarem o processo de cura clínica, não necessitando de tratamento ${ }^{6,7}$.

O objetivo desse artigo é relatar o caso clínico de um paciente, apresentando lesão ulcerada em região da face, após identificação da doença através da cadeia polimesare. $\mathrm{O}$ tratamento clinico foi realizado a aplicação intralesional de glucantime nas lesões tipicamente compatíveis a úlcera em região da face.

\section{MÉTOdOS}

Trata-se de um estudo de caso, com a descrição do diagnóstico e evolução da do- ença de um paciente. Elucidando os mecanismos da doença e dos tratamentos, a partir de estudos clínicos e laboratoriais, visto que traz grande relevância e contribuição cientifica, traz o tratamento de uma paciente com diagnostico de Leishmaniose, uma patologia clínica que representa um desafio no tratamento, por se tratar de úlceras formadas na região da face, além da gravidade e deformidade inerentes a evolução da mesma. É feito o relato desde a primeira consulta, como protocolo utilizado e acompanhamento do processo de cura. A pesquisa foi realizada nos anos de 2019 e 2020 com duração de 1 ano e 05 meses.

Foi adotado o método através de fontes bibliográficas a partir de artigos científicos, livros e protocolos fornecidos pelo Ministério da Saúde (MS), revistas científicas tais como: Revista Acta Tropica, Revista da Sociedade Brasileira de Medicina Tropical, Jornal de Medicina Tropical e Higiene, Revista Saúde Coletiva de Barueri, dentre outros.

A coleta dos dados foi realizada na Unidade Básica de Saúde Gentil Carneiro, localizada no município de Rorainópolis - Roraima, através do prontuário médico do paciente, J. S. S, 34 anos, que autorizou a publicação dos seus dados clínicos como também fotografias para uso exclusivo de pesquisa cientifica através da assinatura do Termo Livre de Consentimento (TLC) fundamentado nas Normas e Diretrizes Brasileiras que ordenam as pesquisas envolvendo seres humanos, incluindo as Resoluções n ${ }^{\circ} 466 / 12$ do Conselho Nacional de Saúde - CNS.

Como critério de inclusão foi utilizado a confirmação através de exame laboratorial a patologia Leishmaniose Cutânea e a exposição de úlceras faciais. A primeira consulta médica ambulatorial ocorreu 


\section{artigo}

Wanderley, P.L.V.; Veloso, C.E.T.; Monção, R.V.;

Tratamento na atenção básica de leishmaniose cutânea com aplição intralesional de glucantime em região da face

Figura 01 - Lesão na face iniciando o tratamento com a aplicação da primeira dose.

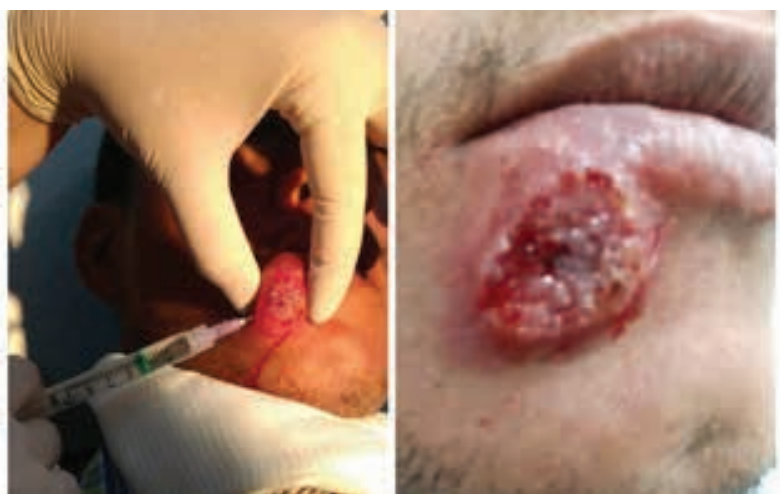

Fonte: Os pesquisadores, 2019.

Figura 02 - lesão na face apresentando primeiros sinais de evolução durante tratamento

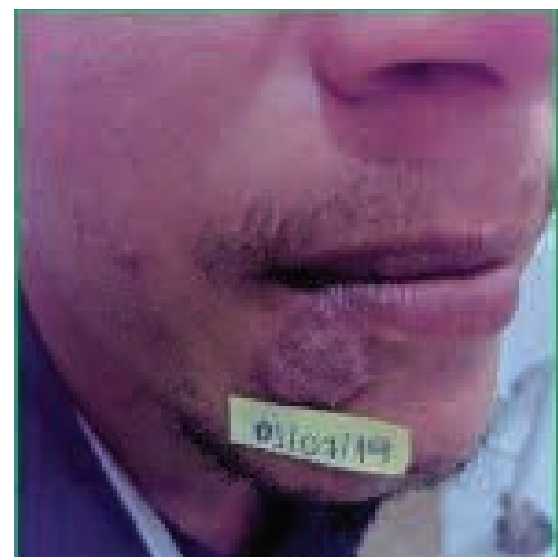

Fonte: Os pesquisadores, 2019.

Figura 03 - Evolução da lesão na face após a segunda aplicação do tratamento intralesional.

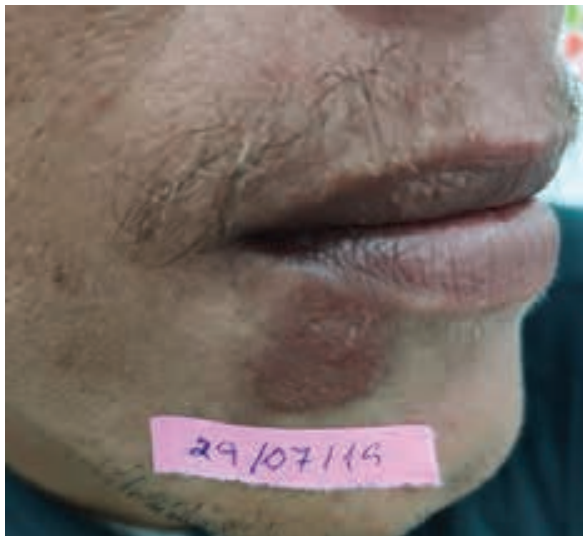

Fonte: Os pesquisadores, 2019. em 03 de junho de 2019, queixando-se de feriada em região da face, perioral, há 2 meses, havendo crescimento da lesão com o decorrer do tempo, sem resposta a tratamento para patologia fúngicas e bacteriana, na investigação da história social, foi constado que o mesmo havia estado em região de garimpo há 4 meses, desde então a lesão começou pequena e foi crescendo, com suspeita de Leishmaniose, foi solicitado a biopsia através do exame de raspagem de lesão e pesquisa, e o mesmo veio positivo.

\section{RESULTADOS}

O tratamento tradicional foi apresentado, glucantime 3 ampolas intravenosa por 20 dias, o total de 60 ampolas para todo o tratamento, porém o mesmo referiu a dificuldade social de comparecer ao hospital, devido morar em zona Rural e não ter transporte para vir a cidade fazer tratamento diário durante os 20 dias, então foi sugerido pela médica da unidade o tratamento intralesional, com infiltração de glucantime em local da lesão, cada aplicação seria com intervalo de 15 dias, e avaliação da lesão para ver se necessitava de outra aplicação, o controle, seria com fotos e exame físico do paciente. Antes da primeira aplicação foram solicitados exames como eletrocardiograma, função hepática, renal e hemograma.

Não foram vistas nenhuma alteração no exame que justificasse a contraindicação ao tratamento, então a primeira aplicação foi realizada 17 de junho de 2019 na ferida de aproximadamente $1,5 \mathrm{~cm}$ por $1,8 \mathrm{~cm}$ perioral , com aspecto de borda crostosa , fundo limpo, porem infiltrativo, foi feito assepsia local com clorexedina degermante, infiltração de anestesia local-lidocaína, e posterior infiltração de glucantime $2,5 \mathrm{ml}$ em lesão, foi infiltrado o suficiente para deixar o aspecto tumeficado, não houve intercorrências com o procedimento. Na figura 01 podemos observar a lesão incialmente.

A segunda aplicação ocorreu em 01 de julho de 2019, o paciente compareceu referindo que esteve bem, porém logo após 
a primeira aplicação o mesmo relatou que teve turvor da visão e vertigem que durou poucos minutos, sem outras intercorrências. A lesão se apresentava menor, com início de epitelização, foi feito assepsia, anestesia local e posterior aplicação de glucantime $1 \mathrm{ml}$ até a lesão ter o aspecto elevado, após cerca de 10 minutos após aplicação paciente relatou tuvor da visão e vertigem, com curta duração, cerca de 2 minutos o episódio, sem mais intercorrências. Na figura 02 podemos acompanhar a evolução primária do paciente.

A terceira e última aplicação foi realizada em 29 de julho de 2019 , o paciente compareceu referindo que esteve bem desde última aplicação, não apresentou ne- nhum sintoma após ser liberado para casa e esse retorno, a lesão já se encontrava menor que última aplicação, com a extensão quase toda epitelizada, foi feito $1,5 \mathrm{ml}$ de glucantime, sem intercorrências. Na figura 03 pode-se ver a evolução da lesão.

Paciente foi orientado a voltar a unidade com frequência de 15 dias para acompanhar epitelização da lesão, após 15 dias paciente retornou à unidade básica e foi constatado que a lesão se encontrava bem menor e totalmente epitelizada. No 6 retorno paciente já se encontrava sem lesão aparente, paciente foi acompanhado por um período de 1 ano após aplicações, onde constatou-se que lesão não teve reeicidiva e teve cicatrização total. Na figura 04 ve-

Figura 04 - lesão cicatrizada após tratamento em um período de 6 meses.

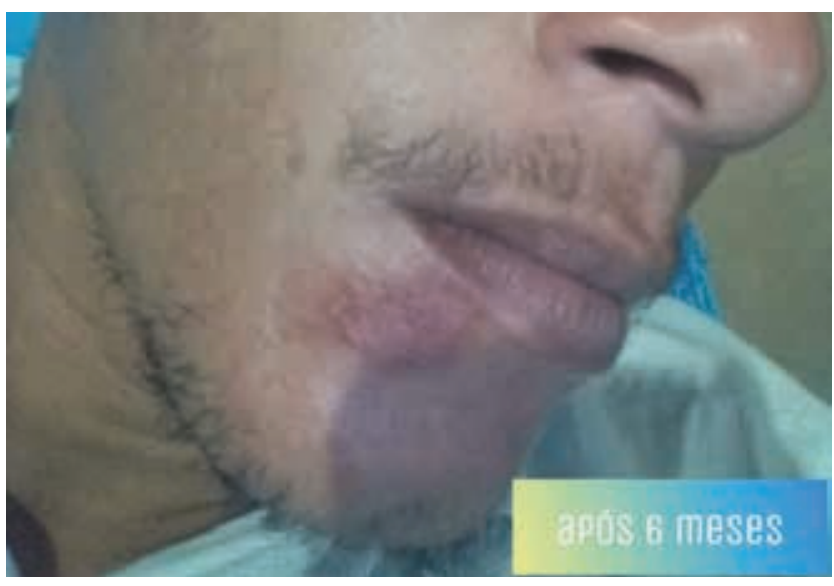

Fonte: Os pesquisadores, 2019.

Figura 05 - paciente sem vestígios da lesão na face.

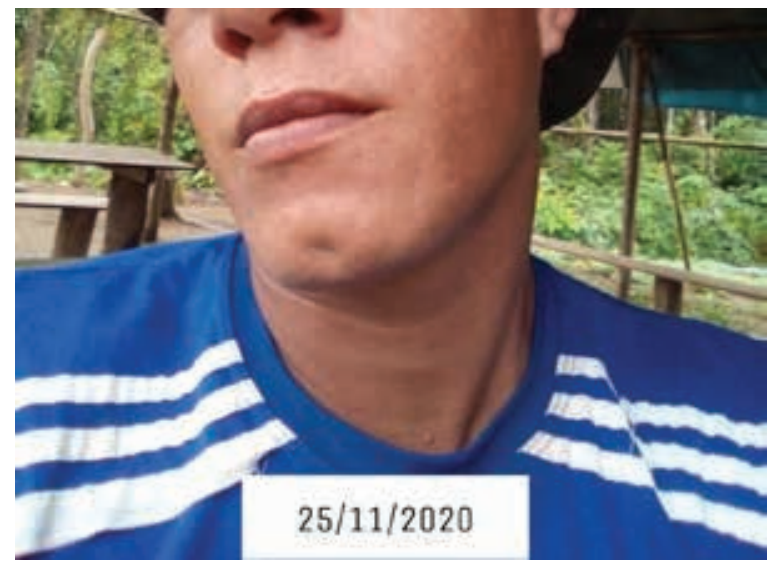

Fonte: Os pesquisadores, 2019. mos a lesão totalmente cicatrizada após tratamento.

Sendo assim, o tratamento total foi usado cerca de 2 ampolas e meia, um contraste grande quando se analisa o tratamento INTRAVENOSO, menos efeito colateral do mesmo. Na figura 05 vemos o paciente sem nenhum vestígio da lesão após um período aproximadamente de um ano e meio.

\section{DISCUSSÃO}

No Brasil, o tratamento tradicional da leishmaniose cutânea, o fármaco de primeira escolha para o tratamento é o antimoniato de N-metil-glucamina (Glucantime) (AM), recomendado pelo Ministério da Saúde, na dose de 10 mg-20 mg Sb5+/ $\mathrm{kg} /$ dia durante vinte dias consecutivos para Leishmaniose Cutânea. Pode haver resposta ao uso dessa terapêutica, entretanto recidivas são comuns8. Em comparação com o tratamento convencional que ultiliza cerca de 60 ampolas intravenoso, foram utilizadas apenas 2,5 ampolas intralesionais

O Método intralesional foi liberado recentemente pelo Ministério da Saúde, no ano de 2017, com algumas restrições: lesão única, $<3 \mathrm{~cm}$, não recomendado em regiões periarticular e cabeça9 ${ }^{9}$ Apesar de ser um método já descrito como eficaz e benéfico em várias partes do corpo, ainda não há estudos descrevendo a eficácia do tratamento intralesional em lesões na face.

Vale ressaltar que durante o tratamento o relato do paciente a cerca de um efeito colateral, a vertigem, associado a droga glucantime, porém efeito leve e pouco duradouro em relação ao efeito colateral do intravenoso, que são mais graves e intensos, decorrendo de a droga ser antiga e com muito efeito colateral, e quando usada em grande quantidade possuem alta toxicidade, podendo trazer riscos para o paciente $^{10}$.

Devido a grande quantidade de efeitos colaterais que a droga possui é recomendado que se faça o uso das ampolas intravenoso em ambiente hospitalar. o que acaba inviabilizando o seu uso em locais de difí- 


\section{artigo}

cil acesso a saúde, onde os postos de saúde são $\mathrm{km}$ de distância do paciente, onde são justamente as áreas de maior foco da doença, região de endemia, região amazônica, onde a saúde é precária e de difícil acesso a população de risco ${ }^{11}$.

$\mathrm{O}$ método intralesional veio para revolucionar a saúde local, onde a saúde é precária e de difícil acesso, pois teve sua eficácia comprovada com eficiência e rapidez utilizando uma quantidade menor da droga obtendo um resultado de desaparecimento TOTAL da lesão ${ }^{12,13}$.

\section{CONCLUSÃO}

O presente estudo traz a percepção da gravidade da Leishmaniose, hoje categorizada no grupo de doenças negligenciadas, devido ao pouco investimento neste cam- po em questão gerando poucos estudos de drogas eficientes com menos efeitos colaterais, ressaltando que é uma doença grave, crônica, que leva a incapacidade e interfere na vida social do paciente portador da patologia, por isso ressalta-se a importância de um diagnóstico e tratamento precoce.

Tendo em vista que o objetivo da pesquisa visou o tratamento da lesão ulcerada em região da face, através da identificação pela cadeia polimesare, faz-se necessária levando a solução para áreas de difícil acesso, onde a saúde é precária, e não há hospitais, apenas unidades de saúde, o método intralesional veio para revolucionar o tratamento devido ao uso de menor quantidade de ampolas, menos efeitos colaterais, além de haver pouco abandono do tratamento e recidiva. Recomenda-se ainda o exame dermatológico/otorrino- laringológico periódico desse paciente, para descartar qualquer possibilidade de recidiva, bem como orientações claras aos pacientes sobre os sinais e sintomas das lesões mucosas.

Com isso conclui-se que a atuação da conduta médica nesse caso foi imprescindível, uma vez o tratamento teve que ser mudado devido a falta da medicação para o tratamento tradicional, e assim corroborar para a conclusão de diagnóstico e planejamento da terapêutica empregando a recomendação do tratamento clínico foi realizado a aplicação intralesional de glucantime nas lesões tipicamente compatíveis a úlcera em região da face. Levando em consideração a fundamental importância da qualidade de vida social do paciente evitando cicatrizações desfigurantes e/ ou mutilantes.

\section{REFERÊNCIAS}

1. Gontijo, B et al. American cutaneous leishmaniasis. Revista da Sociedade Brasileira de Medicina Tropical 36(1):71-80, jan-fev, 2003.

2. Costa, J.M.L. Epidemiology of the Leishmaniasis in Brazil: Gaz. méd. Bahia 2005; 75:1(JanJun):3-17. Acesso em 05 de julho de 2017. Disponível em:

3. Brasil. Ministério da Saúde. Secretaria de Vigilância em Saúde. Manual de Vigilância da Leishmaniose Tegumentar Americana. Ministério da Saúde, Secretaria de Vigilância em Saúde. - 2. ed. atual. - Brasília: Editora do Ministério da Saúde, 2010. 180 p.: il. - (Série A. Normas e Manuais Técnicos). Acesso em 02 de junho de 2017. Disponível em:

4. Lima MVN, Oliveira RZ, Lima AP, et al. Leishmaniose cutânea com desfecho fatal durante o tratamento com antimonial pentavalente. An Bras Dermatol. 2007; 82 : 269-271. doi: 10.1590 / S0365-05962007000300010.

5. Organização Mundial da Saúde. Relatório Técnico Série 949. Controle da leishmaniose. Genebra, 2010, 186 p.

6. Marsden PD, Tada MS, Barreto AC, Cuba CC. Cicatrização espontânea de úlceras cutâneas de Leishmania braziliensis braziliensis. Trans R Soc Trop Med Hyg. 1984; 78 : 561-562. doi: 10.1016 / 0035-9203 (84) 90087-7.

7. Costa JML, Vale KC, França F, et al. Cura espontânea da leishmaniose causada por Leishmania viannia braziliensis em lesões cutâneas. Rev Soc Bras Med Trop. 1990; 23 : 205-208. doi: 10.1590 / S0037-86821990000400004

8. Lopes, Antônio Carlos. Tratado de clínica médica, volume 2 / Antônio Carlos Lopes. 3. ed. Rio de Janeiro: Roca, 2016.
9. Brasil. Ministério da Saúde. Secretaria de Vigilância em Saúde. Departamento de Vigilância Epidemiológica. Manual de recomendações para diagnóstico, tratamento e acompanhamento de pacientes com a coinfecção Leishmania-HIV / Ministério da Saúde, Secretaria de Vigilância em Saúde, Departamento de Vigilância Epidemiológica - Brasília: Ministério da Saúde, 2011.

10. Lucia, RNBP. Distribuição espaço temporal dos casos humanos de leishmaniose tegumentar americana notificados no estado do rio de janeiro de 2001 a 2013 e associação com variáveis clínicas e populacionais. Ministério da Saúde. 2016, Rio de Janeiro.

11. Vasconcellos, E.de C., Pimentel M. I., Schubach A. de O., de Oliveira R. de V, Azeredo-Coutinho R. B., Silva F. da C., Salgueiro M. de M., et all. Short Report: Intralesional Meglumine Antimoniate for Treatment of Cutaneous Leishmaniasis Patients with Contraindication to Systemic Therapy from Rio de Janeiro (2000 to 2006). The American Journal of Tropical Medicine and Hygiene , 31 de julho de 2012, 87 (2): 257-260.

12. De Oliveira Duque, M. C.; Quintão Silva, J. J.; Soares, P. A. O.; Magalhães, R. S.; Horta, A. P. A.; Paes, L. R. B.; et all. Comparison between systemic and intralesional meglumine antimoniate therapy in a primary health care unit. ACTA TROPICA , v. 193, p. 176-182, 2019.

13. De Souza Abreu, M., Macedo Torquato de Siqueira, J. M. ., Cleves da Silva Maia, J. ., Barguil Nepomuceno, D. ., Barros Araújo Lopes Luz, E. ., \& Ferreira Mendes-Sousa, A. . (2021). Aspectos epidemiológicos e distribuição espacial da leishmaniose visceral em Picos, Piauí, Brasil . Saúde Coletiva (Barueri), 11(65), 5846-5857. https://doi.org/10.36489/saudecoletiva.2021v11i65p5846-5857 